\title{
Silicon Nanotips Antireflection Surface for Micro Sun Sensor
}

\author{
Sam Y. Bae ${ }^{1}$, Choonsup Lee ${ }^{2 *}$, Sohrab Mobasser ${ }^{1}$ and Harish Manohara ${ }^{1}$ \\ ${ }^{1}$ Jet propulsion Laboratory, 4800 Oak Grove Drive, Pasadena CA 91106 \\ ${ }^{2}$ California Institute of Technology, 1200 California Blvd., Pasadena CA 91125 \\ "CHOONSUP.LEE@ .JPL.NASA.GOV
}

\begin{abstract}
We have developed a new technique to fabricate antireflection surface using silicon nano-tips for use on a micro sun sensor for Mars rovers. We have achieved randomly distributed nano-tips of radius spanning from $20 \mathrm{~nm}$ to $100 \mathrm{~nm}$ and aspect ratio of 200 using a two-step dry etching process. The $30^{\circ}$ specular reflectance at the target wavelength of $1 \mu \mathrm{m}$ is only about $0.09 \%$, nearly three orders of magnitude lower than that of bare silicon, and the hemispherical reflectance is $\sim 8 \%$. By changing the density and aspect ratio of these nanotips, the change in reflectance is demonstrated. Using surfaces covered with these nano-tips, the critical problem of ghost images that are caused by multiple internal reflections in a micro sun sensor was solved.
\end{abstract}

Keywords-antireflection, nano-tips, Nanotechnology, Mars

\section{INTRODUCTION}

NASA's planetary surface exploration missions that have landed on the surface of Mars included one or more sun sensors. The sun sensors have been used to determine the direction of a rover or to establish 3-axis attitude information to point a high gain antenna towards the earth. At the Jet Propulsion Laboratory, a novel Micro Sun Sensor (MSS) is being developed and flight qualified for future Mars missions [1-3]. The MSS is a miniaturized pinhole camera consisting of a mask, a spacer, and a focal plane. The focal plane is an Active Pixel Sensor (APS) chip and the optics is a piece of silicon wafer coated on one side with thin gold layer perforated by an array of pinholes of $\sim 50-\mu \mathrm{m}$ diameter. This serves the purpose of a mask, and is mounted on a spacer $1000 \mu \mathrm{m}$ from the APS making the system a pinhole camera. The sun rays penetrate silicon mask through the pinholes and form an image on the APS [1-3]. Based on the location of the image on the APS, the MSS determines sun angles that can be used to compute location coordinates with respect to the sun. However, this set up suffers from multiple internal reflections from the focal plane onto the back of the reflective goldcoated mask, and back on to the focal plane causing ghost images, which severely limits the accuracy of the MSS [3]. This problem can be corrected by employing an anti-reflective surface on the back of the mask.

Up to now, many different ways to make antireflection surfaces have been reported [4-8]. For example, porous silicon surface has been used as an antireflection surface [4]. But, it is very easily damaged. A subwavelength structure (SWS) with a surface grating period smaller than the light wavelength has been used for an antireflection surface [5-6], but this requires an expensive fabrication process. Surface texturing with a low aspect ratio is another technique to reduce reflection [7-8]. However, it is well known that achieving a deeper tapered shape offers a better suppression of reflection [5].

Using a two-step dry etch process, H. Manohara has reported a fabrication technique to produce high aspect ration silicon nano-tips in NASA Tech Brief [9]. The greatest advantage of the process is that it is inexpensive, simple, and can be used on large area substrates. The process has inherent capability to be easily integrated with any monolithic silicon fabrication schemes [9]. In this work, we have adapted this technique of microfabricating high aspect ratio nano-tips to create highly efficient antireflection surface without using nanolithography. In this paper, we will describe the antireflection surface fabrication technique and the optical characteristics of the nano-tips surface.

\section{NANO-TIPS FORMATION}

The basic principle of the fabrication technique is to grow micro-etch masks, which can then be etched away along with the substrate to make sharp tips (for the sake of clarity from here on the first step will be referred to as the RIE-step and the second step will be referred to as the DRIE-step). In the RIEstep, a cleaned silicon substrate, either with or without a pattern, is subjected to reactive ion etching process with carbon tetrafluoride and oxygen mixture of $96 \%$ to $8 \%$ respectively at an RF power of $200 \mathrm{~W}$ for a certain predetermined time. This process causes the growth of a certain fluorine-based compound on the substrate in randomly distributed sites. These growths look like short, approximately quadrilateral-cross sectional stumps with a concave top, as shown in Figure 1 (a)-(d)-left. These are familiarly known as the "polymer RIE grass," an unwanted by-product of the dry etching process [10]. Depending on the process time these growths sites may vary in their side dimension from $100 \mathrm{~nm}$ to $500 \mathrm{~nm}$ and grow as tall as a few hundred $\mathrm{nm}$. The density of these sites decreases inversely with the process time. This is obvious as the neighboring sites merge due to growth as the process time is increased. This can be seen in Figures 1 (a)(d)-left, which shows the increasing size of the micro etch 
masks as the process time increases from 5 minutes to 40 minutes. Energy dispersive X-ray spectra (EDX) revealed a high concentration of fluorine in these growth sites, as shown in Figure 2.

In the next step, the substrate with the randomly distributed micro-etch masks is subjected to a deep reactive ion etching process (DRIE), which uses alternate cycles of etching and passivation. The etch gas is sulfur hexafluoride $\left(\mathrm{SF}_{6}\right)$ and the passivation gas is Octafluorocyclobutane $\left(\mathrm{C}_{4} \mathrm{~F}_{8}\right)$. The gas flows of $\mathrm{SF}_{6}$ and $\mathrm{C}_{4} \mathrm{~F}_{8}$ are $130 \mathrm{sccm}$, and $85 \mathrm{sccm}$, respectively. The coil power and platen power are $600 \mathrm{~W}, 140 \mathrm{~W}$, respectively. The "polymer grass" grown in the RIE-step acts as an etch mask in this step slowing down the etch rate directly underneath the mask. This after 8 minutes process time causes dramatic-looking, tall silicon spike-like structures with sharp tips of $15 \mathrm{~nm}$ to $20-\mathrm{nm}$ diameter, as shown in Figures 1 (a)(d)). Because of the DRIE process, these tips exhibit scalloping along their height, which gives them the corkscrewlike appearance. Depending on the thickness of the micro-etch masks, these tips can be fabricated to heights in excess of 20 micrometers. An interesting point to be noted here is that the final nanotip density varies inversely as the micro-etch mask density from the RIE-step. This is because an optimum thickness of the mask is required to successfully achieve sharp tips in the DRIE process (because even the etch mask itself gets etched during DRIE, but at a much slower rate). Such thickness of the mask is less likely to be achieved in shorter RIE runs. So, the thin micro-etch masks get completely etched away during DRIE resulting in ridged and pitted silicon surface, as shown in Figure 1 (a)-right and vice-versa as shown in Figure 1(d)-right. Figure 3 shows the enlarged view of high density nano-tips with the inset showing the close-up of one of the tips itself of diameter $\sim 20 \mathrm{~nm}$.
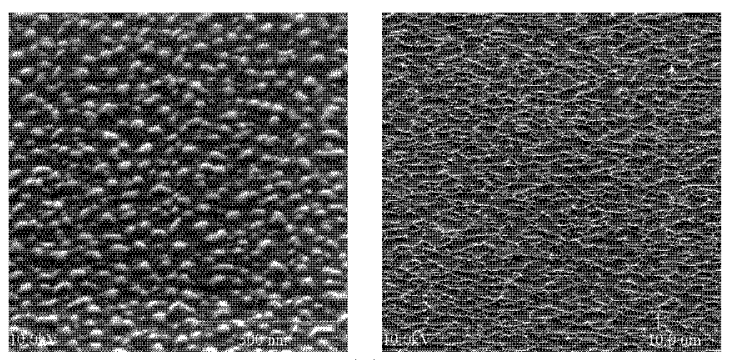

(a)
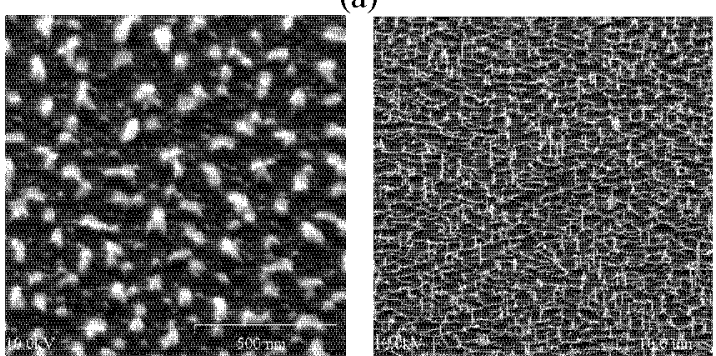

(b)
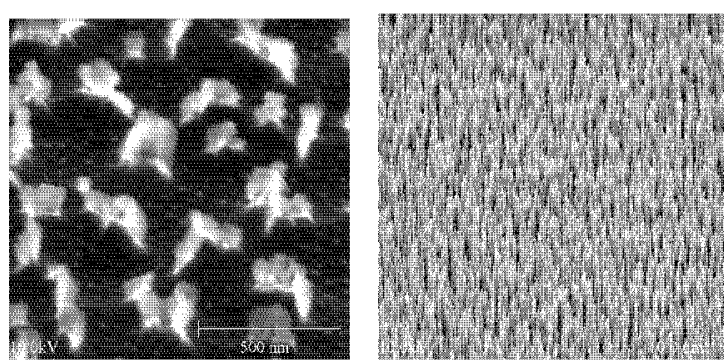

(c)
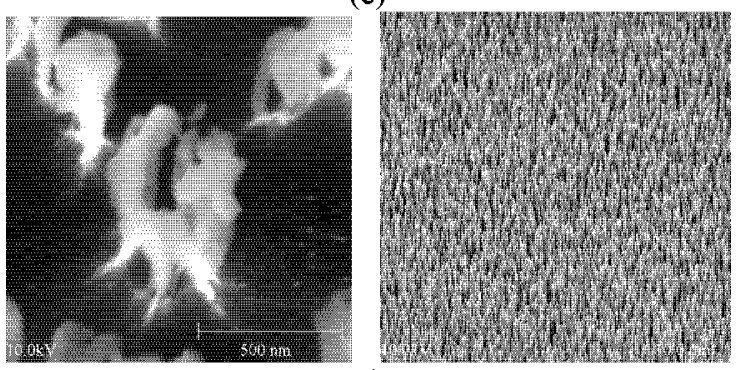

(d)

Figure 1. SEM micrographs depicting the density test results. Left: (a)-(d) show polymer grass (micro-etch masks) grown for $5 \mathrm{~min}, 10 \mathrm{~min}, 20 \mathrm{~min}$ and $40 \mathrm{~min}$ on degenerately doped Si substrate in a $\mathrm{CF}_{4}$ RIE process. Right: (a)-(d) show corresponding silicon nanotips produced from a subsequent DRIE process (total etch time of 8 minutes for each sample using $\mathrm{SF}_{6}$ etching and $\mathrm{C}_{4} \mathrm{~F}_{8}$ passivation cycles). Notice the inverse dependence of Si nano-tip density w.r.t. polymer micro-etch mask density.

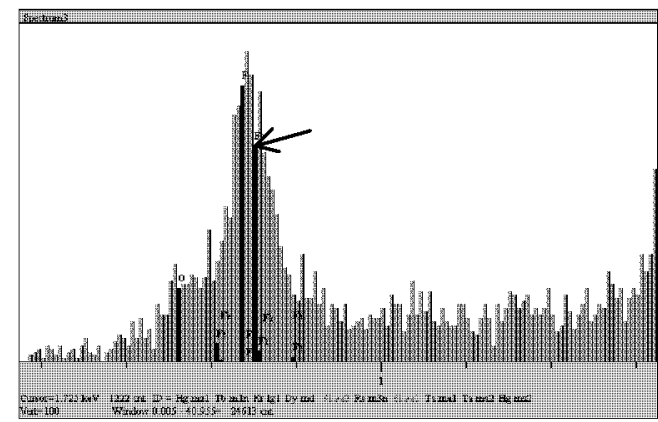

Figure 2. EDX spectra of the RIE grass. The relative intensities are high for fluorine indicating a fluorine predominant compound.

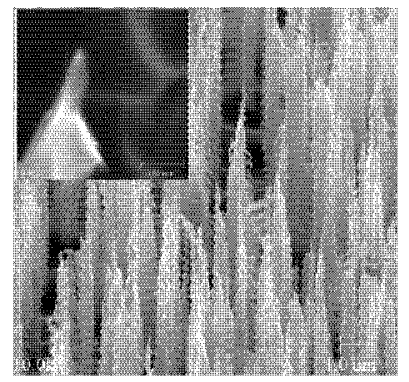

Figure 3. A magnified view of high-density, high-aspect ratio corkscrew-like silicon nanotips. The inset shows close-up of one of the tips with an estimated tip diameter of $\sim 20 \mathrm{~nm}$. 


\section{SUN SENSOR MASK FABRICATION}

Figure 4 shows the fabrication procedures for sun sensor mask. First, photolithography is performed to define the aperture array on a silicon substrate and polymer "RIE-grass" is grown on it, as shown in Figure 4 (a) [10]. Second, the substrate, with these randomly distributed features, is subjected to a DRIE process, as shown in Figure 4 (b) to create high-aspect-ratio silicon spikes with sharp tips. Third, $\mathrm{Cr} / \mathrm{Au}$ is evaporated on the nano-tips surface to absorb the infrared light except at the apertures, as shown in Figure 4 (c). At the end the photoresist is removed by dipping the substrate into acetone and a layer of $\mathrm{Cr}$ is evaporated on the backside of the substrate for the attenuation of sunlight, as shown in Figure 4 (d). A point to be noted here is that the RIE grass growth tends to be less dense in the immediate vicinity of the patterned PR within a margin of 1 to $2 \mu \mathrm{m}$ around the pattern. This sometimes causes no nano-tip formation just in that vicinity of the pattern. However, we have not seen this to cause any effect on the antireflection performance of the coating on the sun sensor mask.

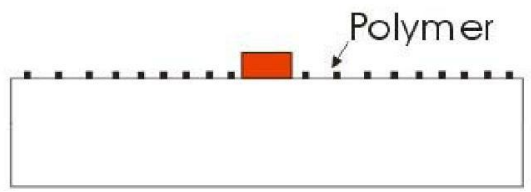

(a)

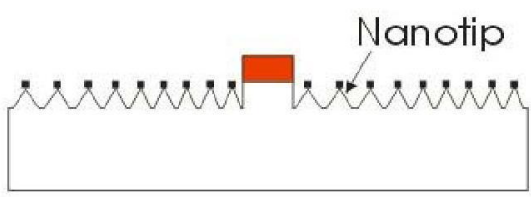

(b)

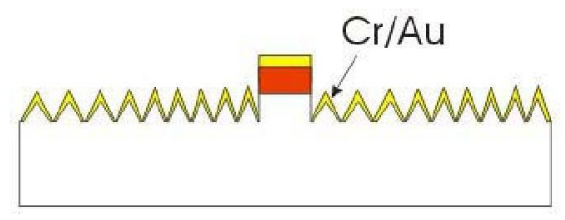

(c)

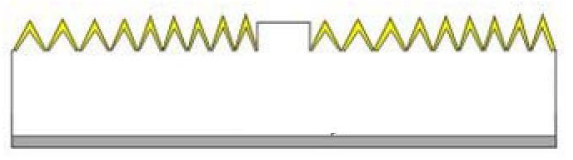

(d)

Figure 4. The sun sensor mask fabrication procedure. (a) Photoresist (PR) has been patterned to define the aperture array and "RIE-grass" has been grown on the silicon substrate. The density of the "RIE-grass" is determined by both the RF power and the process time. (b) Nano-tips have resulted from the DRIE because "RIE-grass" works as an etch mask. (c) $\mathrm{Cr} / \mathrm{Au}$ has been evaporated on the nano-tips to increase absorption of the infrared sunlight. (d) PR has been removed to form apertures and $\mathrm{Cr}$ has been deposited on the backside of the substrate to attenuate the light intensity.

\section{RESULTS AND DISCUSSIONS}

Figure 5 shows the photograph of the $\mathrm{Cr} / \mathrm{Au}$-coated silicon substrate and $\mathrm{Cr} / \mathrm{Au}$-coated nano-tips substrate. The nano-tips substrate is almost black due to the extremely small reflectance. Figure 6 shows the fabricated high-aspect-ratio nano-tips of the antireflection surface. This functional nanopatterning is achieved without using nanolithography. A desirable feature of the resulting aperture array is that it continues to work as an attitude sensor in a situation where one or more apertures are blocked by Mars dust, as shown in Figure 6 (a). Figure 6 (b) shows a single aperture surrounded by the nano-tips. The aperture has $\mathrm{Cr}$ on the top surface as the light attenuator. The light incident on the aperture will pass through the silicon and make an image on the Active Pixel Sensor (APS) chip. The light incident on the nanotips from either side will be absorbed in the metals on the nano-tips. Thus, it can neither form an image on APS chip nor contribute to ghost images via reflection $[1,2]$. The location on the APS of the resulting high contrast images of the aperture array is used to determine the sun angles.

Figure 7 shows the 30 degree specular reflectance measurement data of the nano-tips coated with $\mathrm{Cr} / \mathrm{Au}$. The purpose of the metal here is to improve absorption of the infrared light. It was measured on samples relative to aluminum mirror from 0.3 - 2.5 microns using a Cary 5000 UV-Vis-NIR spectrometer. The samples were next measured using the same attachment relative to $\mathrm{Au}$ from 2.5 to 200 microns using a Bruker $66 \mathrm{~V}$ FTIR with a beam splitter change for the Far IR (16-200 microns), because Al has more absorbance than gold in the Far IR. The 30 degree specular reflectance of the $40 \mathrm{~min} \mathrm{RIE}$ silicon nano-tips with $\mathrm{Cr} / \mathrm{Au}$ at the target wavelength of $1 \mu \mathrm{m}$ is about $0.09 \%$. The same value for bare silicon is almost $35 \%$ at the same wavelength indicating $\mathrm{a} \sim 400$-fold reduction in reflectance by the antireflection surface made of nano-tips coated with $\mathrm{Cr} / \mathrm{Au}$. In addition, as the RIE times increases, the specular reflectance decreases due to the higher density of the nano-tips. The reflectance of the substrate can be adjusted by changing the density of the nano-tips, which is determined by the "RIEgrass" growth process as shown in the figure (the reflectance increases from 40-min RIE surface to 5-min RIE surface as $0.09 \%$ to $38.79 \%$ ). In order to assess the total reflectance from the nano-tip covered surfaces we measured the hemispherical reflectance from $0.3 \mu \mathrm{m}$ to $2.5 \mu \mathrm{m}$ wavelength range, which covers the wavelength of interest. Figure 8 shows the total hemispherical reflectance of the samples with three different nano-tip densities (corresponding to RIE times) as before. These measurements were done using the Cary 5000 UV-Vis-NIR spectrometer with a Cary integrating sphere attachment. The samples were measured relative to a spectralon (Teflon) reflectance standard from 0.3 to 2.5 microns. Once again, it can be seen that the 40-min RIE surface has the lowest hemispherical reflectance among the three measured, with $\sim 8 \%$ at $1 \mu \mathrm{m}$ wavelength. The same for 10-min and 5-min surfaces are $27.17 \%$ and $86.83 \%$, respectively. 


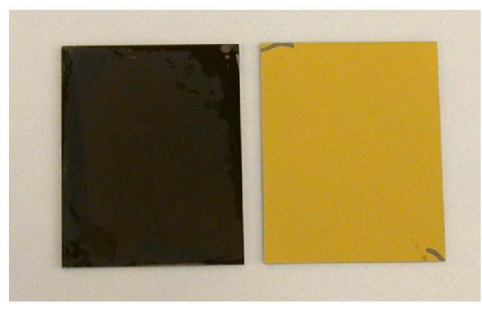

Figure 5. Appearance to naked eye of the fabricated surfaces in standard laboratory light. Photographs of the antireflection nano-tips substrate coated with $\mathrm{Cr} / \mathrm{Au}$ (left side) and bare silicon substrate coated with $\mathrm{Cr} / \mathrm{Au}$ (right side). Notice the dramatic change in appearance because of extremely small reflectance of the nano-tips substrate that makes them look almost black. The sample sizes are $2.2 \mathrm{~cm}$ by $2.8 \mathrm{~cm}$

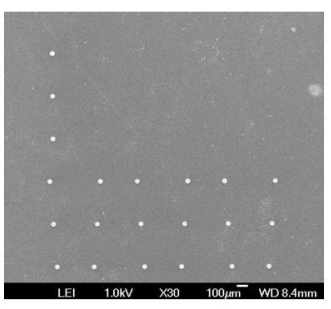

(a)

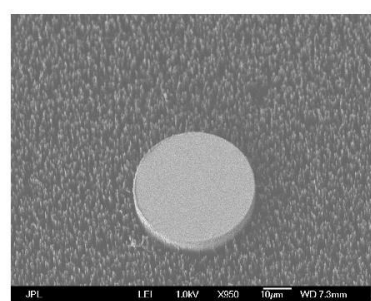

(b)
Figure 6. SEM micrographs of an aperture array. All images were taken at a $20^{\circ}$ tilt. (a) The complete aperture array. This array can continue to work as an attitude sensor in a situation where one or more apertures are blocked by Mars dust. (b) High magnification of one aperture of height $\sim 20 \mu \mathrm{m}$. The diameter of an aperture is $\sim 50 \mu \mathrm{m}$.

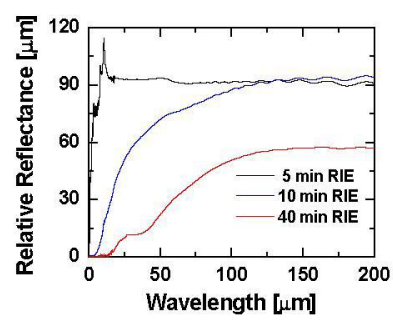

(a)

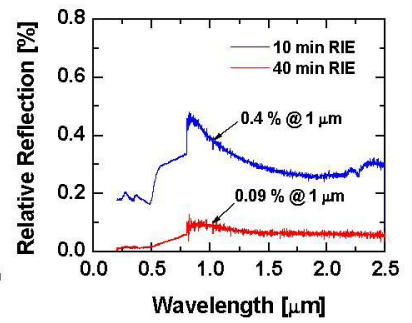

(b)
Figure 7. Specular reflectance of silicon nano-tips at $30^{\circ}$ angle showing, (a) the relative reflectance of $5 \mathrm{~min}, 10 \mathrm{~min}$, and 40 min RIE samples from 0 to $200 \mu \mathrm{m}$ wavelength range, (b) magnified section of the reflectance graph showing details of the relative reflectance in the short wavelength- 0 to $2.5 \mu \mathrm{m}$. At $1 \mu \mathrm{m}$ target wavelength, the reflectance of 40-min surface is about $0.09 \%$.

\section{CONCULSIONS}

We have successfully demonstrated a novel antireflection surface with extremely low reflectance using randomly distributed silicon nanotips. The fabrication process of these nano-tips uses standard silicon fabrication methods and is conducive for integration with batch-fabricated silicon devices. By changing the RIE process times, the density and aspect ratios of nano-tips can be varied and hence the reflectance. We have achieved typical nano-tip radius spanning from $20 \mathrm{~nm}$ to $100 \mathrm{~nm}$ with an aspect ratio of 200 without using nanolithography. The specular and the total hemispherical reflectance from the dense 40-min RIE nano-tip surface were measured to be $0.09 \%$ (relative to an aluminum surface) and $\sim 8 \%$ (relative to a standard spectralon surface) respectively at a target wavelength of $1 \mu \mathrm{m}$. This reflectance is nearly three orders of magnitude lower than that of plain silicon. This process has been used in the micro sun sensor for Mars rovers to remove ghost images that, otherwise, drastically decrease the accuracy of these sensors. The process developed here is reproducible from the performance point of view. That is, the nano-tip formation itself is a random process as a result of which, it is impossible to produce nano-tips in the same identical location from sample to sample. However, qualitatively, the surface density of these tips is reproducible from sample to sample, which has been evident from the reflectance data of multiple samples. For example, among three different 40-min samples tested, the measured $30^{\circ}$ specular reflectance is in the range of $0.05 \%$ to $0.09 \%$.

\section{ACKNOWLEDGMENT}

The research described in this paper was carried out by the Jet propulsion Laboratory, California Institute of Technology under a contract with the National Aeronautics and Space Administration. The authors would like to thank Dr. Mark S Anderson at the JPL for the reflectance measurements.

\section{REFERENCES}

[1] C. C. Liebe; S. Mobasser, Aerospace Conference, 2001, IEEE Proceedings. Vol. 3, 2001, pp. 1565-1572. [2] S. Mobasser; C. C. Liebe, 2003 IEEE Conference on Control Applications, June 23-25, 2003.[3] S. Mobasser; C. C. Liebe; J. Naegle; Choonsup Lee, $2^{\text {nd }}$ International Conference on Recent Advances in Space Technologies (RAST) 2005, June 09-11.[4] R. Bilyalov; L. Stalmans; J. Poortmans, Joumal of Electrochemical Society, 150 (3), pp. G216, 2003. [5] Y. Kananori; K. Hane; H. Sai; H. Yugami, Applied Physics Letters 78(2), pp. 142, 2001.[6] K. Hadobas; S. Kirsch; A. Carl; M. Acet; E. F. Wassermann, Nanotechnology 11 pp. 161 2000. [7] G. Kumaravelu; M. M. Alkaisi; A. Bittar, IEEE Photovoltaic Specialists Conference 2002 Conference Record of the Twenty-Ninth IEEE, pp. 258. [8] M. Schnell; R. Ludenmann; S. Schaefer, IEEE Photovoltaic Specialists Conference 2000 Conference Record of the Twenty-Ninth IEEE, pp. 367. [9] H. Manohara, NASA Tech Briefs, vol. 28, no. 11, pp. 62, November 2004. [10] Trion Technical Papers - "Cook Book" - RIE Grass, http://www.triontech.com/techpapers/RIEgrass.html 